\title{
BIAS Word inventory for work and employment diversity, (in)equality and inclusivity (Version 1.0)
}

\author{
Alla Konnikov (University of Alberta), Nicole Denier* (University of Alberta), Yang Hu* \\ (Lancaster University), Karen D. Hughes (University of Alberta), Jabir Alshehabi Al-Ani \\ (University of Essex), Lei Ding (University of Alberta), Irina Rets (Lancaster University), \\ Monideepa Tarafdar (University of Massachusetts Amherst) \\ * Corresponding authors \\ Nicole Denier, Department of Sociology, University of Alberta, nicole.denier@ualberta.ca \\ Yang Hu, Department of Sociology, Lancaster University, yang.hu@lancaster.ac.uk
}

\begin{abstract}
The language used in job advertisements contains explicit and implicit cues, which signal employers' preferences for candidates of certain ascribed characteristics, such as gender and ethnicity/race. To capture such biases in language use, existing word inventories have focused predominantly on gender and are based on general perceptions of the 'masculine' or 'feminine' orientations of specific words and socio-psychological understandings of 'agentic' and 'communal' traits. Nevertheless, these approaches are limited to gender and they do not consider the specific contexts in which the language is used. To address these limitations, we have developed the first comprehensive word inventory for work and employment diversity, (in)equality, and inclusivity that builds on a number of conceptual and methodological innovations. The BIAS Word Inventory was developed as part of our work in an international, interdisciplinary project - BIAS: Responsible AI for Labour Market Equality - in Canada and the United Kingdom (UK). Conceptually, we rely on a sociological approach that is attuned to various documented causes and correlates of inequalities related to gender, sexuality, ethnicity/race, immigration and family statuses in the labour market context. Methodologically, we rely on 'expert' coding of actual job advertisements in Canada and the UK, as well as iterative cycles of inter-rater verification. Our inventory is particularly suited for studying labour market inequalities, as it reflects the language used to describe job postings, and the inventory takes account of cues at various dimensions, including explicit and implicit cues associated with gender, ethnicity, citizenship and immigration statuses, role specifications, equality, equity and inclusivity policies and pledges, work-family policies, and workplace context.
\end{abstract}

KEYWORDS: Bias; Canada; diversity; equity; gender; inequality; inventory; inclusivity; UK; wording. 


\section{INTRODUCTION}

As labour market processes become increasingly digitalised with the use of online job postings and algorithms, there is growing evidence that the deployment of artificial intelligence (AI) contributes to the perpetuation of gender and racial biases in hiring, promotion, and career progression (Ajunwa 2020). These developments raise significant concerns over the potential impact of AI for ongoing efforts to improve work-related equity, diversity, and inclusion (EDI) ${ }^{1}$ in contemporary economies. While the question of how bias enters into organizational and labour market processes is a complex one, it is clear from past research that the language used in job advertisements plays a central role. Now classic studies, such as Bem and Bem (1973) show how gendered words in job advertisements discourage applicants of the opposite sex from applying for jobs. Subsequent studies have also shown how implicit gender preferences are signalled through the use of specific traits and behavioural terms (Askehave and Zethsen 2014).

In this paper, we offer theoretical, methodological, and substantive insights to advance research on this issue. Specifically, we introduce and outline the development of the BIAS word inventory for capturing equity, diversity, and inclusivity in labour market processes, which emerges from an international, interdisciplinary project - BIAS: Responsible AI for Labour Market Equality - in Canada and the United Kingdom (UK). ${ }^{2}$ To contextualize our contribution, we begin Section 2 with a brief critical review of existing word inventories, noting their strengths but also the limitations of social and cognitive psychological perspectives that inform much work in this area. We also outline our own sociological approach that is attuned to context and the social construction of gender, sexuality, ethnicity/race as they relate to inequalities in the labour market. In Section 3, we discuss our methodological approach which relies on 'expert' coding of actual job advertisements in Canada and the UK, and iterative cycles of inter-rater comparison an approach that differs from established methodologies based on public perceptions of masculinity or femininity of specific words. Following a 'toolkit' approach in Section 4, we introduce our word inventory which offers comprehensive coverage of EDI related words that may reflect employers' preferences with respect to the gender, racial/ethnic, and citizenship characteristics of potential applicants. Section 5 provides technical notes for using the word inventory. Section 6 concludes with a discussion of the implications and future directions of our research.

Please note that we will provide regular updates to our word inventory in order to reflect changing workplace organisation, policies, identity categories, and sociocultural dynamics. Please refer to the version history shown at the end of the article title for a record of our updates.

\footnotetext{
${ }^{1}$ For consistency, we use the terminology 'equity, diversity and inclusivity' (EDI) in this paper as it is well established in Canada and the UK, the two countries where our research is carried out. Other terms and acronyms that are used in other countries include diversity and inclusion (D\&I) and diversity, equality, and inclusion (DEI).

${ }^{2}$ Further information about the BIAS project can be found here.
} 


\section{EXISTING INVENTORIES AND OUR APPROACH}

There are two dominant approaches to developing inventories of biased language. The first is rooted in psychological understandings of sex/gender roles and personality traits and identifies words traditionally associated with masculine or feminine characteristics (i.e. caregiving, assertiveness; agentic vs. communal) (Cryan et al. 2020; Wille \& Derous 2018). The Bem SexRole Inventory, for instance, is a list of words associated with masculinity and femininity identified through studies where participants indicated words or traits that are desirable for men or women (Gaucher, Frisen, \& Kay 2011; Bem 1974; Tang et al. 2017). Further research has demonstrated the importance of such word choices for attracting or deterring applicants to certain jobs, and that gender-biased language varies according to the level of male dominance in an occupation or level of gender inequality in a country (Gaucher, Frisen, \& Kay 2011; Hodel et al. 2017). The second approach relies on examining the linkages between gendered names, pronouns and nouns, and language, often using a large corpus to identify word associations (Gonen \& Goldberg 2019). Recently, scholars have begun to integrate these methods. For instance, Cryan et al. (2020) combine lexicon-based and word association approaches, first identifying target words, then using crowdsourced participants to score thousands of words for masculinity and femininity, and finally using supervised learning to identify additional biased words in a larger corpus.

While these approaches are useful in conceptualizing and identifying biased language, they are necessarily limited. First, both approaches thus far have been used to focus on a singular dimension of inequality, most often gender. Second, both offer a 'one size fits all' list of words, failing to account for differences in language used in different social settings. For instance, the contents of a word inventory may not be exhaustive of the ways employers subtly signal gender preferences or preferences related to other employee identities such as ethnicity, race, sexuality and immigration status. Yet, corpus-based approaches highlight biased text across a generic range of contexts (e.g. the Wikipedia) such that identified biased words might not be locally meaningful, especially in formalised and regulated labour markets. Together, both fail to explicitly attend to contextual cues in language. These gaps motivate our proposed method.

The method we outline below builds upon and goes beyond psychological approaches to identify contextually relevant social cues in job postings that may signal preferences for certain types of workers. We rely on expert coding conceptually informed by research in sociology, labour markets, and management studies. By relying on expert qualitative coding rather than mere empirical associations of words and typical gender roles, we are also able to identify concepts and words linked to other forms of categorical inequality, such as those based on race, ethnicity, immigration status, and sexual orientation. There are well-established separate bodies

of research on the labour market outcomes of women, ethnic/racial/visible minorities, and 
immigrants that point to unique barriers and some shared challenges in the labour market. Our approach is attuned to how job postings may convey these unique and shared challenges, as discussed in further detail below.

\section{METHODS}

Our analysis is informed by qualitative coding methodology - the process of labelling and mapping the meanings and messages in text (Hsieh \& Shannon 2005). The deductive nature of qualitative coding facilitates an in-depth and embedded understanding of the messages conveyed in the text (Saldaña 2021). These meanings are grounded within the specific context in which these texts are produced and read (Krippendorff 2004). Our analysis of the cues that appear in the job postings is grounded in our expertise as social scientists and scholarship on labour market inequality connected to gender, ethnicity, race, immigration and other dimensions. A similar approach of relying on academic literature for generating word embeddings was reported in Manzini et al. (2020).

Informed by the conceptual framework outlined above, our methodology for producing a word inventory for identifying biased wording in job postings comprised several steps, including preliminary qualitative coding, conceptual analysis of the corpus of preliminary codes, and intercoder verification and finalisation. First, we derived a randomly-selected sample of 160 real job postings from the UK and Canada databases used in the multidisciplinary project - BIAS: Responsible AI for Labour Market Equality. Part of this project focuses on analysing, understanding, and mitigating the use of biased language in job advertisements. Using the sample, the team, building on their disciplinary expertise in employment and organization, immigration, racial/ethnic relations, gender, work and family, conducted a preliminary qualitative content analysis to identify cues associated with gender, ethnicity, citizenship, workfamily balance, and Equity Diversity and Inclusion (EDI) policies. Next, the team performed a conceptual analysis of the preliminary coding, grounded in the labour market inequality literature. The final phase included an inter-rater reliability verification and close collaboration

with computer scientists to refine the corpus and adapt it to algorithmic use. At this stage, words (or word roots) that have extremely low frequencies of appearance (less than 20 counts) in the large database of over 26 million job postings were eliminated from the inventory. The next sections elaborate on each of the steps in detail.

\subsection{Preliminary qualitative coding}

In the BIAS database, the total numbers of job postings are 22,069,704 in the UK and 4,363,743 in Canada, spanning the time period 2018-2020. From this database, a random sample of 160 real-data job postings from Canada and the UK was generated using the Mersenne Twister 
random function in Python (Matsumoto \& Nishimura 1998). Each of the first four authors manually coded 40 of the selected job postings (20 from Canada and 20 from the UK) to identify all potential cues related to gender, sexuality, ethnicity/race, citizenship/immigration status, and (in)equality, diversity, and inclusion. This preliminary coding generated a large corpus of keywords that clustered across three main dimensions: 1) explicit gender cues (such as pronouns [she/he/her/him]) and ethnicity/race/citizenship cues (such as Black, Asian, etc.); 2) implicit psychological, behavioural and cognitive cues that describe candidates and roles, such as agentic and communal traits; and 3) explicit and implicit cues that describe the workplace characteristics and work(-family) and EDI policies.

\subsection{Conceptual analysis of the preliminary corpus}

The next stage involved organising the large pool of keywords generated from the preliminary coding across distinct conceptual dimensions and categories. During this phase, we conducted a multi-stage content analysis. First, we cleaned and de-duplicated the cues identified by different coders. Second, we conducted another round of coding to identify the orientations of the keywords within its conceptual cluster, such as 'masculine', 'feminine' and 'neutral' for gender cues and cues that 'promote' versus 'hinder' diversity based on ethnicity/race/citizenship. This stage was informed by the scholarship on gender inequality, work-family relations, racial/ethnic relations and immigration, and contextualised within the existing definitions and policies of EDI in Canada and the UK. While during the process of identifying gender cues we partly relied on previously documented agentic and communal psychological traits (Bem 1974; Gaucher, Frisen, \& Kay 2011), the analysis of cues in other dimensions such as ethnicity/race/citizenship and work-family and EDI policies was entirely novel.

To perform the conceptual analysis of dimensions other than cognitive gender cues, we turned attention to traits typically associated with status-based forms of (de)valuation as well as explicit barriers, policies, or programs that may hinder or encourage certain applicants. For example, we incorporated words describing skills, such as 'communicational' and 'soft' skills following evidence that the notion of soft skills can lead to the exclusion of immigrants and ethnic minorities in the Canadian labour market (Guo 2009; Shan 2013). In another example, we identified words that replicate documented structural barriers that exclude women from the maledominated fields, such as 'long hours' and 'frequent trips' (Cha 2013; Reid, O’Neill \& Blair-Loy 2013; Lewis-Enright et al. 2009). In this stage, to ensure the reliability and validity of this analysis, we combined both separate and shared iterations of analyses between the four key coders, followed by discussions until a consensus on the final conceptual dimensions was reached. 


\subsection{Inter-rater reliability, inventory cleaning and finalisation}

To ensure the scientific rigour of our word inventory, we paid particular attention to inter-rater validity and reliability. Specifically, each phase included an individual coding of data conducted by four independent coders specialising in labour market inequalities associated with gender and sexual orientation; work and family; immigration and ethnic relations. In each round of coding, individual coding was followed by group sharing and discussion of the preliminary outcomes among the four coders. This combination of individual and group analysis allowed the team to trace the development of the word inventory, to ensure inter-rater reliability in each phase and contextualise each phase within relevant scholarly literature, policies and definitions. As a key purpose of the development of this word inventory was to ensure accurate computational analysis of language bias in job postings, the word inventory was finalised with assistance from computational experts. This step included reworking the words into their 'root' version to allow identification of multiple words with the same root. The final version of the word inventory is in the format suitable for the use in word embeddings' techniques such as Word Embeddings Association Test (WEAT) and debiasing word/sentence embedding.

\section{THE BIAS WORD INVENTORY}

In this section, we report the word inventory generated from our research. The inventory is formed, at the first level, of two broad sets of words - i.e. gender cues and diversity cues related to traits such as race and ethnicity, immigration status, citizenship and so on. While inventories for the identification of gender biases have had a long tradition (for example, dating back to Bem's inventory developed in 1974), we particularly separate out the second set of diversity cues that have been less systematically identified and synthesised in existing research.

On the one hand, within the set of gender cues, we further distinguished four lists of words in terms of psychological cues, role description, work-family characteristics, and social characteristics. Detailed information on and theoretical underpinnings for each word list are presented below. Here, it is important to note that we removed from our gender inventory the words that overlap with those in the Gaucher, Freizen and Kay (2011) and Bem (1974) inventories (see links at the end of the article for these inventories). Thus, if our inventory is used to identify psychological cues, the inventory needs to be used in combination with the Gaucher, Freizen and Kay (2011) and Bem (1974) inventories - the links to which are provided at the end of this article. On the other hand, the diversity cues cover three lists of words in terms of social characteristics (e.g. ethnic and racial status, citizenship, etc.), policy and legislation cues, and organisational culture and practice.

Together, the word lists combine to form a comprehensive word inventory for work and employment diversity, (in)equality and inclusivity. But each specific word list can be used on its 
own to probe different dimensions of labour market, human resources, and organisation processes. To optimise our word inventory for computation analyses, we use the symbol '*' to indicate the cut-off for word root, and multiple database formats are provided.

\subsection{Gender}

\subsubsection{Psychological cues (excluding the Gaucher et al. [2011] and Bem [1974] inventories)}

The first list builds on existing, widely used psychological inventories of traits associated with gender roles. Gender inequality in labour markets is long-standing and despite changing gender relations, legislation, and economic dynamics, progress towards gender equality has largely been characterised as 'stalled' (Cotter, Hermsen, \& Vanneman 2004; England, Levine, \& Mishel 2020). Although women's labour force participation has increased dramatically in the last thirty years, women have made only limited and selective inroads into traditionally male-dominated occupations and face wage penalties even in highly paid occupations, contributing to persistent gender segregation and gender wage gaps (Adams 2010; England, Levine, \& Mishel 2020; Sohrab, Karambayya \& Burke 2011). Status-based expectations attached to gender form the standards against which people are selected, evaluated, and rewarded, which may ultimately contribute to both segregation and gender wage disparities (Ridgeway 2014). For instance, women are often perceived as less committed to professional careers (Wallace 2008) and less suitable to perform tasks in fields that have been traditionally male-dominated (Hatmaker 2013). These expectations and organizational priorities shape employer, colleague, and customer perceptions of an 'ideal worker' (Acker 1990). The list of words below captures psychological cues that tap into these gendered expectations.

\section{Feminine}

accura*; attentive; caring;

collaborative;

committed; creative; dedicated; diploma*; follow*; friendly; organized; patient; person*centered; persua*; polite; thoughtful;

\section{Masculine}

accountab*; alone; authoritative; best; busy; calm*; can-do; communicat*; composed; confiden*; detail; driven/self-driven; dynamic; eager; effective*; efficient*; eloquent; empower; energ*; engag*; enthusias*; exceed; excel*; exceptional; exciting; firm; forward thinking; fun; hard-working; high quality; initiative; innovative; inspirational; limitless; motivat*; outgoing; outstanding; passion*; practical; pragmatic; proactive; problem solv*; productiv*; reliab*; resilien*; resolve;

\section{Neutral ${ }^{3}$}

adapt*; authentic; availab*; clear; concise; courteous; curious; delightful; easy; engaged; fair; flexib*; frank; good; happy; help*; integrity; objective; open*; perseverance; persistent; planning; professionally; punctual; qualified; reassur*; respect*;

\footnotetext{
${ }^{3}$ We are expanding the neutral word lists in our next version update. Please refer to the up-to-date version of the article for a fuller list of neutral words. It is worth noting that neutral words are less relevant for bias identification, but they can be used in bias mitigation (e.g. replacing biased words with neutral ones).
} 
welcome

resourcefulness; respected; self-motivated; self-

starter; serious; strong; tactful; talented;

timekeeping; time* management; vibrant; winning responsive; rewarding; tenacious*; think outside the box; trustworthy

\subsubsection{Role description ${ }^{4}$}

The second list focuses on the roles employers expect job applicants to carry out at work. The work practices expected of a job candidate can often be gendered. The importance of role enactment in (re)producing gender (biases) is firmly anchored in the conceptualisation that gender, as an achieved social status, is enacted through the ongoing 'doing' of gender (West \& Zimmerman 1987). In the literature on gender in the labour market, it has long been established that different work tasks and occupational contexts are often sex-typed to denote gendered traits and preferences (Cook 1994; Fagenson 1990; Luhaorg \& Zivian 1985). The following list of words capture potentially gendered ways in which employers describe the expected roles of a worker.

\section{Feminine}

administrat* (skills);

listening (skills);

organizational

(skills); people

skills; social skills;

soft skills

\section{Masculine}

physical*; bend*; challeng*; crouch*; demand*; driving; exert*; fit*; hands on; heavy load*; lift*; kneel*; mov*; negotiat*; numera*; pressure; pulling; pushing; reaching; risk*; safety precautions; speed; stamina; stoop*; stress*; transport; twisting; weight handling

\section{Neutral}

clean drivers; cold (manufacturing environment); driver's license; humid (manufacturing environment); literate; multitask*; repetitive; secure work; standing; walking; wet (manufacturing environment)

\subsubsection{Work-family characteristics}

Gender biases at work are constructed in relation to other life domains such as the family. Indeed, gender biases in job advertisements are often associated with the differential roles women and men assume in different-sex families. Decades of work-family scholarship clearly shows that employers' family(-friendly) policies have a significant impact on the gendered labour force participation of job candidates and employees (Kelly et al. 2014; Moen 2018; Sweet 2013). Gender biases and inequalities in phenomena such as the female marriage penalty, fatherhood premium, and motherhood penalty in the labour market all reflect the importance of the work-family interface in shaping labour market dynamics and outcomes (Fuller \& Qian 2021; Killewald 2012; Qian \& Hu 2021; Weisshaar 2018). Further, as expected roles in the family differ for individuals of different gender identities, experiences of work-family conflict

\footnotetext{
${ }^{4}$ This list does not include gendered occupational titles, such as waiter/waitress.
} 
and balance also vary across different genders (Chung \& Van der Lippe 2020). As a result, employers' flexible work, work-family balance, and family support policies, and the temporal regimes of employment schedules play significant roles in shaping work participation and experiences in highly gendered ways (Allen et al. 2012; Collins 2020; Thompson et al. 1999). The following list includes potential cues that may invoke gendered work-family orientations and considerations.

\section{Feminine}

5 days per week; 8.00 am to $6.00 \mathrm{pm}$; bonus; child*; childcare vouchers; commission package; contracted (hours); family friendly; family values; flexib*; flexible benefits; flex* (+ e.g. time, location, etc.); guaranteed Hours; maternity (leave); Monday to Friday; parental (leave); part time; paternity (leave); permanent; regular hours; relocation package; remote work; scheme; standard hours; statutory sick pay; work/life balance

\section{Masculine}

international (travel); travel; additional hours; after hours; call outs; different areas; different locations; evenings; fixed term; holiday cover; live-in; location change; multisite; night (shifts); on-site visits; overtime; sickness cover; standby; temporary; urgen*; waking nights; weekends; willing* to travel

\subsubsection{Social characteristics}

In addition to roles, traits, and skills, job advertisements may include direct references to gender, through explicit mentions of gendered nouns, pronouns, and identity markers.

\section{Feminine}

gal; *women; *woman; lady; her; she

\section{Masculine}

guy; *men, *man; his; he

\section{Neutral}

accommodation; commission; fulltime; global opportunities; hours of work; rota schedule; shift*

\subsection{Diversity}

We also address issues of diversity, building on research on labour market discrimination and efforts to improve equity, diversity, and inclusion (EDI) in the workplace. Our analysis of job postings generated three-word lists relating to: 1) the social characteristics of workers (e.g. immigrant/citizenship, race/ethnicity, disability, sexuality, etc.); 2) policies and legislation; and 3) organizational culture and practice. Overall, we identified three types of cues (i.e. prodiversity, exclusionary, and neutral) although all three types are present only in relation to the social characteristics of workers, while the word lists on policies and legislation, and organizational culture and practice, reflect 'pro-diversity' cues exclusively. 


\subsubsection{Social characteristics (immigration/citizenship; race/ethnicity; sexual orientation; disability)}

The first list (below) on social characteristics draws on wide-ranging evidence of discrimination and unequal outcomes in relation to race/ethnicity, immigrant/citizenship status, sexual orientation, disabilities and other characteristics of workers. Studies of ethnic and/or racial differences in labour market outcomes, for instance, confirm pervasive discrimination in relation to hiring, earnings, and promotion (Banerjee et al. 2018; Hirschman \& Garbes 2021; Pedulla \& Pager 2019; Quillian et al. 2017; Zschirnt \& Ruedin 2016). Immigrants also face barriers due to the non-recognition of foreign credentials (Reitz 2001; Guo \& Shan 2013; Reitz, Curtis \& Elrick 2014), and discrimination based on language, religion, and skin tone (Banerjee 2009; Creese \& Wiebe 2012). A growing body of evidence also confirms discriminatory processes on the basis of sexual orientation (Denier \& Waite 2019; Drydakis 2015; Mize 2016) and disability (Foster \& Wass 2013; Maroto \& Pettinicchio 2015).

\begin{abstract}
Pro-diversity cues
Immigration / Citizenship

bilingual*; Mandarin; French; second

language

Ethnicity / Race

B(A)ME; Black; Asian; ethnic;

minority; visible minorities; Indigenous;

Aboriginal; FNIHB; BIPOC

Disability

disab*

General

under-represent* (groups)

\begin{tabular}{|c|c|}
\hline & \\
\hline Immigration / Citizenship & Immigration / \\
\hline authorized (to work); birth & Citizenship \\
\hline certificate; British (citizen); & outside the EU; EU/EEA \\
\hline Canadian (citizen); culturally- & (non); Resident Labour \\
\hline appropriate; eligible (to work); & Market Test; UK Visas \\
\hline $\begin{array}{l}\text { English; EU (citizen); immigr*; } \\
\text { legally; National Insurance }\end{array}$ & $\begin{array}{l}\text { and Immigration; } \\
\text { foreign national; work }\end{array}$ \\
\hline Number; passport; permanent & permit; Immigration \\
\hline resident; police check; residence & Act; fluency duty; \\
\hline (UK/EU/Canada); right (to work); & LMIA (Labour market \\
\hline Social Insurance Number; veteran* & impact assessment) \\
\hline
\end{tabular}

Exclusion cues

Immigration/Citizenship

certificate; British (citizen);

Canadian (citizen); culturally-

English; EU (citizen); immigr*;

legally; National Insurance

Number; passport; permanen

(UK/EU/Canada); right (to work);

Social Insurance Number; veteran*

\author{
Neutral \\ outside the EU; EU/EEA \\ (non); Resident Labour \\ and Immigration; \\ foreign national; work \\ permit; Immigration \\ Act; fluency duty; \\ impact assessment)
}

Immigration /

\subsubsection{Policy and legislation cues}

As labour market inequality and discriminatory practices come under growing scrutiny, normative pressure, broader social movements, legislation and regulatory oversight, employers have sought to boost the presence and inclusion of 'historically underrepresented' groups. This can be seen in a range of EDI pledges, policies and initiatives, as well as pro-diversity language, that seeks to attract specific groups of workers and/or emphasize the value of cultural diversity (Koellen 2021; Konrad et al. 2006; Triana et al. 2021; Yadav \& Lenka 2020).
\end{abstract}




\section{Pro-diversity cues related to policy and legislation}

Aboriginal; accessibl*; all genders; all-qualified; APEP; B(A)ME; barrier-free; disab*; Disabilities Act 2005; discriminat*; divers*; Employment Equity; encourages* (members); equal opportunity; equal*; Equality Act 2010; equit*; fair; FNIHBs; free from* (discrimination/harassment); harassment; human rights; mpartiality; inclusi*; Indigenous; Investment Industry Regulatory Organization of Canada (IIROC); language*; LGBT+; LGBTQ+; Merit*; minority candidates; nationalities; neutrality; Privacy Information Statement Protected characteristics Protection and Electronic Documents Act ("PIPEDA"); required by law; Stonewall; under-represent*; Work Act 1974

\subsubsection{Organizational culture and practice}

Beyond using language aimed at attracting specific groups of workers and emphasizing the value placed on diversity, employers also highlight the positive aspects of workplace culture and practices generally and in relation to EDI (Broadbridge \& Fielden, 2018; Konrad et al., 2006; Kossek \& Lee, 2020).

\section{Pro-diversity cues related to organizational culture and practice}

accessible (location); advance careers; annual leave; award-winning [team]; benefits package; best; best people; best places; busy; care; career advancement; career development; career progression; central values; challenging; committed; community; compensation; competitive pension; culture; dynamic; employee assistance; empower; fast-paced; friendly; fun; global; good relations; growth; health care account; ideal location; innovative; international; internationally recognised; investment and saving programs; leading; life assurance; limitless; mission; multi-site; multidisciplinary; paid benefits; personal accident insurance; personal development; pressure; professional development; progression opportunities; progressive; rewarding; sociable; staff discount; support; supportive; team; tenure; training; training courses; transport links; vibrant; young.

\section{TECHNICAL USE OF THE WORD INVENTORY}

One of the applications of the inventory is to assist computational analysis of word embeddings performed for the project, BIAS: Responsible AI for Labour Market Equality, which aims in part to identify and mitigate gender- and ethnic-related bias in job postings' language (Ding et al 2022; Hu et al 2022). The word inventory can be adapted for use in a number of computational techniques: 1) Natural Language Processing (NLP) methods, where word embeddings are numerical representations of words' relations in the text (Zhao et al. 2018) and 2) Word Embeddings Association Tests (WEAT) that produce the association difference between two sets of words (Caliskan et al. 2017). Previous evaluation methods relied on general word inventories that were not designed specifically for the labour market context and therefore, the algorithms 
trained on such data may not be accurate in capturing context-specific biases in labour market processes. Our word inventory helps to overcome this limitation since word embeddings are trained using labour market job text data, which can facilitate more accurate identification of biased language. The advantage of using a context-specific word inventory extends to the process of data debiasing using word embeddings. Most state-of-the-art debiasing methods require a list of biased words in order to extract biased information from the word embeddings. This information is then removed to obtain the debiased version of the word embeddings. Therefore, the quality of the word inventory directly shapes the performance of the debiasing algorithm.

\section{CONCLUSION, IMPLICATIONS AND FUTURE DIRECTIONS}

In this paper, we presented a systematic, interdisciplinary word inventory for capturing equity, diversity and inclusivity (EDI) in the workplace and in labour market processes. Previous research exploring the textual representation of EDI issues in the labour market, with a predominant focus on gender cues, has primarily drawn on the disciplines of social and cognitive psychology. Expanding on this tradition, our word inventory speaks directly to the broader social construction of gender, sexuality, ethnicity/race and immigration status, as well as their attendant inequalities in the labour market. In this context, our inventory also covers textual cues related to work-family policies, immigration regulations and policies, workplace and organisational context, and EDI pledges and policies that directly and indirectly bear on gender, ethnic/racial, and migrant inequalities in the labour market. As a whole, our inventory provides a comprehensive coverage of EDI-related words that may signal employers' gender, sexuality, racial/ethnic, and native-migrant preferences in communicating their version of an ideal worker. The sub-lists included in our inventory can be used separately to probe the presence/absence of textual cues related to gender, ethnic/racial, and migrant biases in distinct dimensions and at distinct stages of labour market processes.

The limitations of our word inventory suggest several important directions for future research and development. First, the researchers involved in the process of manual coding were experienced in social research on EDI issues in the labour market and may thus be more sensitive to EDI-related cues than lay readers. This means that the development of the word inventory is both explicitly informed by existing theories on EDI in the labour market and implicitly informed by the coders' tacit knowledge of the theories. A further step for the development of the inventory is to examine how lay readers, e.g. job seekers and employees, perceive the cues in our inventory in the context of, for example, job advertising and organisational policy communication. This will help add a further layer of triangulation from the reader-reception perspective, in addition to our inter-coder and theoretical validation. 
Second, as the construction of labour market EDI policies and that of EDI-related identities and cues are context-specific, it is important to note that our inventory is developed from a large corpus of job advertisements from the UK and Canada. This means that despite some cross-national generalisability, our inventory is partly shaped by the labour market legislation and broader social and cultural configurations (e.g. pertaining to gender and ethnicity/race) in the two countries. The underpinning corpus is composed of job advertisements - an important site where textual cues relating to gender, sexuality, ethnicity/race, and immigrant status are found. But the extent to which the inventory applies to other corpora, such as organisational regulations and documents in human resources management (e.g. those pertaining to performance evaluation, promotion, retention, etc.) will require further verification. Furthermore, for some important dimensions of inequality, including Indigeneity, disability, and sexuality, our cues focused largely on formal policies related to attracting diverse candidate pools, rather than the well-developed role-based and psychological cues established for gender inequality, an important avenue for further research.

Its limitations notwithstanding, our word inventory can be used in, but is not limited to, the following ways:

- For computer scientists, our word inventory can be used to inform the development of word embeddings and machine learning algorithms, for the development of bias detection and mitigation tools. Traditionally, the detection and mitigation of gender, ethnic/racial, and migrant biases using machine learning and artificial intelligence tended to rely on general-purpose corpora such as Wikipedia and newspaper articles (Brunet et al. 2019; Papakyriakopoulos et al. 2020; Tan \& Celis 2019), which are not well suited to capture EDI related cues in labour market processes in particular and everyday social and organizational life in general. Built on a corpus of job advertisements, our word inventory is particularly suited for identifying cues that are directly related to EDI issues.

- For social scientists researching labour market EDI issues, the inventory (in its totality or its sub-lists) can be used to quantify the extent of presence/absence of gender, sexuality, ethnic/racial and immigrant biases in textual communications in labour market processes (Hu et al. 2022). This can be achieved through a direct word search based on our inventory or through more advanced techniques such as wording embedding and machine learning (Caliskan et al. 2017).

- Our inventory also provides unique opportunities for interdisciplinary research on EDIrelated topics. As our different sub-lists are respectively informed by the disciplines of 
(social) psychology, sociology, social policy, legal studies, and management and organisation studies. Comparative analysis and application of the sub-lists to a given labour market process promises to reveal how the different disciplines can bring to light different aspects of EDI-related dynamics in the labour market.

Finally, it is worth noting that the very conceptualisation of EDI and its related policies and procedures are constantly evolving alongside broader social, political, legal, cultural, and institutional changes, and so should the word inventory. Therefore, the project team will provide regular updates on the inventory using version control. We also welcome feedback and suggestions from our readers to further enrich and refine the inventory.

\section{UPCOMING UPDATES IN THE NEXT VERSION}

The next version update will focus on the following two areas:

1. Provide a more extensive range of neutral words. Such words may not be particularly useful in the context of bias identification, but they will form a key corpus for bias mitigation as users of our inventory use strategies such as word embedding and replacement to reduce or offset biased wordings in a piece of text. See Hu et al. (2022) and Ding et al. (2021) for examples.

2. We will further validate our word inventory using text mining, based on a large, crossnational corpus of more than 26 million job advertisements.

\section{RELEVANT EXISTING WORD INVENTORIES}

1. The Gaucher, Friesen \& Kay (2011) inventory: doi:10.1037/a0022530

2. The Bem (1974) inventory: doi:10.1037/ h0036215

\section{CONTRIBUTION STATEMENT AND ACKNOWLEDGEMENTS}

The first four authors - Konnikov, Denier, Hu, and Hughes - took the lead in developing the word inventory and the first draft of this paper. The remaining authors, listed alphabetically, provided input at various stages of the word inventory development and read and commented on the final manuscript. The team would like to thank Carlota Minero Marquez and Sara Hill for their excellent research assistance.

\section{FUNDING}

This work was supported by the Economic and Social Research Council (ESRC ES/T012382/1) and the Social Sciences and Humanities Research Council (SSHRC 2003-2019-0003) under the 
scheme of the Canada-UK Artificial Intelligence Initiative. The project title is 'BIAS:

Responsible AI for Labour Market Equality'.

\section{REFERENCES}

Acker, J. (1990). Hierarchies, jobs, bodies: A theory of gendered organizations. Gender \& Society, 4(2), 139-158.

Ajunwa, I. (2020). The paradox of automation as anti-bias intervention. Cardozo Law Review, $41(5), 1671-1742$.

Allen, T. D., Johnson, R. C., Kiburz, K. M., \& Shockley, K. M. (2013). Work-family conflict and flexible work arrangements: Deconstructing flexibility. Personnel Psychology, 66(2), $345-376$.

Amatea, E. S., \& Fong, M. L. (1991). The impact of role stressors and personal resources on the stress experience of professional women. Psychology of Women Quarterly, 15(3), 419430.

Askehave, I., \& Zethsen, K. K. (2014). Gendered constructions of leadership in Danish job advertisements. Gender, Work \& Organization, 21(6), 531-545.

Banerjee, R. (2009). Income growth of new immigrants in Canada: Evidence from the survey of labour and income dynamics. Relations Industrielles/Industrial Relations, 64(3), 466488.

Banerjee, R., Reitz, J. G., \& Oreopoulos, P. (2018). Do large employers treat racial minorities more fairly? An analysis of Canadian field experiment data. Canadian Public Policy, $44(1), 1-12$.

Bauder, H. (2003). "Brain Abuse", or the devaluation of immigrant labour in Canada. Antipode, 35(4), 699-717.

Bem, S. L., \& Bem, D. J. (1973). Does sex-biased job advertising “aid and abet" sex discrimination? Journal of Applied Social Psychology, 3(1), 6-18.

Bem, S. L. (1974). The measurement of psychological androgyny. Journal of Consulting and Clinical Psychology, 42(2), 155.

Benard, S., \& Correll, S. J. (2010). Normative discrimination and the motherhood penalty. Gender \& Society, 24(5), 616-646.

Benard, S., Paik, I., \& Correll, S. J. (2007). Cognitive bias and the motherhood penalty. Hastings Law Journal, 59(6), 1359-1388.

Bøler, E. A., Javorcik, B., \& Ulltveit-Moe, K. H. (2018). Working across time zones: Exporters and the gender wage gap. Journal of International Economics, 111, 122-133. 
Broadbridge, A. \& Fielden, S. (Eds.). (2018). Research Handbook of Diversity and Careers. Edward Elgar.

Brunet, M.-E., Alkalay-Houlihan, C., Anderson, A., \& Zemel, R. (2019). Understanding the origins of Bias in word embeddings. Proceedings of the 36th International Conference on Machine Learning Research, 97, 803-811.

Caliskan, A., Bryson, J. J., \& Narayanan, A. (2017). Semantics derived automatically from language corpora contain human-like biases. Science, 356(6334), 183-186.

Carley, K. (1988). Formalizing the social expert's knowledge. Sociological Methods \& Research, 17(2), 165-232.

Cha, Y. (2013). Overwork and the persistence of gender segregation in occupations. Gender \& Society, 27(2), 158-184.

Chiswick, B. R., \& Miller, P. W. (2002). Immigrant earnings: Language skills, linguistic concentrations and the business cycle. Journal of Population Economics, 15(1), 31-57.

Chung, H., \& Van der Lippe, T. (2020). Flexible working, work-life balance, and gender equality: Introduction. Social Indicators Research, 151(2), 365-381.

Collins, C. (2020). Making Motherhood Work: How Women Manage Careers and Caregiving. NJ: Princeton University Press.

Cook, E. P. (1994). Role salience and multiple roles: A gender perspective. The Career Development Quarterly, 43(1), 85-95.

Correll, S. J., Benard, S., \& Paik, I. (2007). Getting a job: Is there a motherhood penalty? American Journal of Sociology, 112(5), 1297-1339.

Creese, G., \& Wiebe, B. (2012). 'Survival employment': Gender and deskilling among African immigrants in Canada. International Migration, 50(5), 56-76.

Denier, N., \& Waite, S. (2019). Sexual orientation at work: Documenting and understanding wage inequality. Sociology Compass, 13(4), e12667.

Ding, L., Yu, D., Xie, J., Guo, W., Hu, S., Liu, M., Kong., L., Dai, H., Bao, Y., \& Jiang, B. (2021). Word embeddings via causal inference: Gender bias reducing and semantic information preserving. Association for the Advancement of Artificial Intelligence (AAAI). arXiv preprint arXiv:2112.05194.

Drydakis, N. (2015). Sexual orientation discrimination in the United Kingdom's labour market: A field experiment. Human Relations, 68(11), 1769-1796.

Fagenson, E. A. (1990). Perceived masculine and feminine attributes examined as a function of individuals' sex and level in the organizational power hierarchy: A test of four theoretical perspectives. Journal of Applied Psychology, 75(2), 204-211. 
Foster, D., \& Wass, V. (2013). Disability in the labour market: an exploration of concepts of the ideal worker and organisational fit that disadvantage employees with impairments. Sociology, 47(4), 705-721.

Frome, P. M., Alfeld, C. J., Eccles, J. S., \& Barber, B. L. (2006). Why don't they want a maledominated job? An investigation of young women who changed their occupational aspirations. Educational Research and Evaluation, 12(4), 359-372.

Frone, M. R. (2003). Work-family balance. In Handbook of Occupational Health Psychology (pp. 143-162). American Psychological Association.

Fuller, S., \& Qian, Y. (2021). Parenthood, gender, and the risks and consequences of job loss. Social Forces. https://doi.org/10.1093/sf/soab078

Gaucher, D., Friesen, J., \& Kay, A. C. (2011). Evidence that gendered wording in job advertisements exists and sustains gender inequality. Journal of Personality and Social Psychology, 101(1), 109-128.

Greenhaus, J. H., \& Allen, T. D. (2011). Work-family balance: A review and extension of the literature. In Handbook of Occupational Health Psychology (pp. 165-184). American Psychological Association.

Guo, S. (2009). Difference, deficiency, and devaluation: Tracing the roots of non-recognition of foreign credentials for immigrant professionals in Canada. Canadian Journal for the Study of Adult Education, 22(1), 37-52.

Guo, S., \& Shan, H. (2013). The politics of recognition: Critical discourse analysis of recent PLAR policies for immigrant professionals in Canada. International Journal of Lifelong Education, 32(4), 464-480.

Higgins, C., Duxbury, L., \& Johnson, K. L. (2000). Part-time work for women: Does it really help balance work and family? Human Resource Management, 39(1), 17-32.

Hirschman, D., \& Garbes, L. (2021). Toward an economic sociology of race. Socio-Economic Review, 19(3), 1171-1199.

Hsieh, H.-F., \& Shannon, S. E. (2005). Three approaches to qualitative content analysis. Qualitative Health Research, 15(9), 1277-1288.

Hu, S., Alshehabi Al-Ani, J., Hughes, D.K, Denier, N., Konnikov, A., Ding, L., Xie, J., Hu, Y., Tarafdar, M. Jiang, B., Kong, L., and Dai, H. (2022). Balancing gender bias in job advertisements with text-level bias mitigation. Frontiers in Big Data, 1-10. doi: 10.3389/fdata.2022.805713

Jännäri, J., Poutanen, S., \& Kovalainen, A. (2018). Gendering expert work and ideal candidacy in Finnish and Estonian job advertisements. Gender in Management: An International Journal, 33(7), 544-560.

Kaneko, M., \& Bollegala, D. (2019). Gender-preserving debiasing for pre-trained word embeddings. arXiv preprint:1906.00742 
Kelly, E. L., Moen, P., Oakes, J. M., Fan, W., Okechukwu, C., Davis, K. D., Hammer, L. B., Kossek, E. E., King, R. B., Hanson, G. C., Mierzwa, F., \& Casper, L. M. (2014). Changing work and work-family conflict: Evidence from the Work, Family, and Health Network. American Sociological Review, 79(3), 485-516.

Killewald, A. (2013). A reconsideration of the fatherhood premium: Marriage, coresidence, biology, and fathers' wages. American Sociological Review, 78(1), 96-116.

Koellen, T. (2021). Diversity management: A critical review and agenda for the future. Journal of Management Inquiry, 30(3), 259-272.

Konrad, A. M., Prasad, P., \& Pringle, J. (Eds.). (2006). Handbook of Workplace Diversity. CA: Sage.

Kossek, E.E. \& Lee, K.H. (Eds.). (2020). Creating Gender-Inclusive Organizations. Toronto: University of Toronto Press.

Krippendorff, K. (2004). Content Analysis: An Introduction to its Methodology (2nd. edition). CA: Sage.

Lewis-Enright, K., Crafford, A., \& Crous, F. (2009). Towards a workplace conducive to the career advancement of women. SA Journal of Industrial Psychology, 35(1), 1-9.

Luhaorg, H., \& Zivian, M. T. (1995). Gender role conflict: The interaction of gender, gender role, and occupation. Sex Roles, 33(9-10), 607-620.

Mahadevan, J., \& Mayer, C.-H. (Eds.). (2017). Muslim Minorities, Workplace Diversity and Reflexive HRM (1st ed.).

Manzini, T., Lim, Y. C., Tsvetkov, Y., \& Black, A. W. (2019). Black is to criminal as caucasian is to police: Detecting and removing multiclass bias in word embeddings. arXiv preprint: 1904.04047.

Maroto, M., \& Pettinicchio, D. (2015). Twenty-five years after the ADA: Situating disability in America's system of stratification. Disability Studies Quarterly, 35(3), 1-34.

Mize, T. D. (2016). Sexual orientation in the labor market. American Sociological Review, 81(6), $1132-1160$.

Moen, P. (2018). It's about time: Couples and careers. NY: Cornell University Press.

Papakyriakopoulos, O., Hegelich, S., Serrano, J. C. M., \& Marco, F. (2020, January). Bias in word embeddings. In Proceedings of the 2020 Conference on Fairness, Accountability, and Transparency (pp. 446-457). https://doi.org/10.1145/ 3351095.3372843

Pedulla, D. S., \& Pager, D. (2019). Race and networks in the job search process. American Sociological Review, 84(6), 983-1012.

Qian, Y., \& Hu, Y. (2021). Couples' changing work patterns in the United Kingdom and the United States during the COVID-19 pandemic. Gender, Work \& Organization, 28(S2), $535-553$. 
Quillian, L., Pager, D., Hexel, O., \& Midtbøen, A. H. (2017). Meta-analysis of field experiments shows no change in racial discrimination in hiring over time. Proceedings of the National Academy of Sciences, 114(41), 10870-10875.

Reid, E. M., O’Neill, O. A., \& Blair-Loy, M. (2018). Masculinity in male-dominated occupations: How teams, time, and tasks shape masculinity contests. Journal of Social Issues, 74(3), 579-606.

Reitz, J. G. (2001). Immigrant skill utilization in the Canadian labour market: Implications of human capital research. Journal of International Migration and Integration, 2(3), $347-$ 378.

Reitz, J. G. (2007). Immigrant employment success in Canada, Part II: Understanding the decline. Journal of International Migration and Integration / Revue de l'integration et de La Migration Internationale, 8(1), 37-62.

Saldaña, J. (2021). The Coding Manual for Qualitative Researchers. London: Sage.

Shan, H. (2013). Skill as a relational construct: hiring practices from the standpoint of Chinese immigrant engineers in Canada. Work, Employment and Society, 27(6), 915-931.

Sweet, S. A. (2013). The Work-Family Interface: An Introduction. CA: Sage.

Tan, Y. C., \& Celis, L. E. (2019). Assessing social and intersectional biases in contextualized word representations. arXiv preprint:1911.01485.

Thompson, C. A., Beauvais, L. L., \& Lyness, K. S. (1999). When work-family benefits are not enough: The influence of work-family culture on benefit utilization, organizational attachment, and work-family conflict. Journal of Vocational Behavior, 54(3), 392-415.

Triana, M. D. C., Gu, P., Chapa, O., Richard, O., \& Colella, A. (2021). Sixty years of discrimination and diversity research in human resource management: A review with suggestions for future research directions. Human Resource Management, 60(1), 145204.

Weisshaar, K. (2018). From opt out to blocked out: The challenges for labor market re-entry after family-related employment lapses. American Sociological Review, 83(1), 34-60.

West, C., \& Zimmerman, D. H. (1987). Doing gender. Gender \& Society, 1(2), 125-151.

Wood, A. J. (2018). Powerful times: Flexible discipline and schedule gifts at work. Work, Employment and Society, 32(6), 1061-1077.

Yadav, S., \& Lenka, U. (2020). Diversity management: A systematic review. Equality, Diversity and Inclusion: An International Journal, 39(8), 901-929.

Zhao, J., Zhou, Y., Li, Z., Wang, W., \& Chang, K.-W. (2018). Learning gender-neutral word embeddings. arXiv preprint:1809.01496. 
Zschirnt, E., \& Ruedin, D. (2016). Ethnic discrimination in hiring decisions: A meta-analysis of correspondence tests 1990-2015. Journal of Ethnic and Migration Studies, 42(7), 11151134. 